\title{
Inclusion of Van der Waals Interactions in DFT using Wannier Functions without empirical parameters
}

\author{
Pier Luigi Silvestrelli ${ }^{1, *}$ and Alberto Ambrosetti ${ }^{1, * *}$ \\ ${ }^{1}$ Dipartimento di Fisica e Astronomia "G. Galilei”, Università di Padova, via Marzolo 8, I-35131, Padova, Italy, and CNR-IOM \\ Democritos, via Bonomea 265, I-34136, Trieste, Italy.
}

\begin{abstract}
We describe a method for including van der Waals (vdW) interactions in Density Functional Theory (DFT) using the Maximally-Localized Wannier functions (MLWFs), which is free from empirical parameters. With respect to the previous DFT/vdW-WF2 version, in the present DFT/vdW-WF2-x approach, the empirical, short-range, damping function is replaced by an estimate of the Pauli exchange repulsion, also obtained by the MLWFs properties. Applications to systems contained in the popular S22 molecular database and to the case of adsorption of Ar on graphite, and Xe and water on graphene, indicate that the new method, besides being more physically founded, also leads to a systematic improvement in the description of systems where vdW interactions play a significant role.
\end{abstract}

\section{Introduction}

Density Functional Theory (DFT) is a well-established computational approach to study the structural and electronic properties of condensed matter systems from first principles. Although current, approximated density functionals allow a quantitative description at much lower computational cost than other first principles methods, they fail[1] to properly describe dispersion interactions. These forces originate from correlated charge oscillations in separate fragments of matter and the leading component (namely, the lowest-order perturbative contribution) is represented by the $R^{-6}$ van der Waals (vdW) interaction[2], due to correlated instantaneous dipole fluctuations. vdW interactions play a fundamental role in determining the structure, stability, and function of a wide variety of systems, including molecules, clusters, proteins, nanostructured materials, molecular solids and liquids, and in adsorption processes of fragments weakly interacting with a substrate ("physisorbed").

In the last few years a variety of practical methods have been proposed to make DFT calculations able to accurately describe vdW effects (for a review, see, for instance, refs. [3-9]). In this respect, a family of such methods, all based on the Maximally Localized Wannier Functions (MLWFs)[10], has been developed, namely the original DFT/vdW-WF scheme[11-13], DFT/vdW-WF2[14] (based on the London expression and taking into account the intrafragment overlap of the MLWFs), DFT/vdWWF2s[15] (including metal-screening corrections), and DFT/vdW-QHO-WF[16] (adopting the coupled Quantum Harmonic Oscillator model), successfully applied to a variety of systems [11-23]: small molecules, water clus-

\footnotetext{
*e-mail: psil@pd.infn.it

**e-mail: ambroset@pd.infn.it
}

ters, graphite and graphene, water layers interacting with graphite, interfacial water on semiconducting substrates, hydrogenated carbon nanotubes, molecular solids, the interaction of rare gases and small molecules with metal surfaces,...

In all these methods a certain degree of empiricism is present since the energetic vdW-correction term is multiplied by a short-range damping function, which is introduced not only to avoid the unphysical divergence of the $\mathrm{vdW}$ correction at small fragment separations, but also to eliminate double countings of correlation effects (in fact standard DFT approaches are able to describe short-range correlations). This damping function contains one or more empirical parameters which are typically set by a trial and error approach or/and are fitted using some reference database.

In a recent paper[24] we have presented a new method, named DFT/vdW-WF2-x, to overcome the above limitation, where the empirical, short-range, damping function is replaced by an estimate of the Pauli exchange repulsion, also obtained by the MLWFs properties. Here we use the DFT/vdW-WF2-x scheme, already successfully applied[24] to the popular S22 benchmark set[25] of weakly interacting molecules and to the case of an Ar atom interacting with graphite, also to describe the adsorption processes of a single $\mathrm{Xe}$ atom and a single water molecule interacting with graphene. Rare gases adsorbed on graphene are interesting since they represent prototypical adsorption systems. Moreover the study of the interactions of graphene with water is very important as a model for the characterization of the interface between water and hydrophobic substrates[26]; in addition, a single water molecule adsorbed on graphene represents a weakly interacting system involving a complex mixture of hydro- 
structure theory. Our results are compared with reference data and confirm that the DFT/vdW-WF2-x method leads to a systematic improvement in the description of systems where vdW interactions play a significant role.

\section{Method}

Here we briefly describe the DFT/vdW-WF2-x method, introduced in ref. [24], where further details can be found. Basically, the electronic charge partitioning is achieved using the Maximally-Localized Wannier Functions (MLWFs), which are obtained from a unitary transformation in the space of the occupied Bloch states, by minimizing the total spread functional[10]:

$$
\Omega=\sum_{n} S_{n}^{2}=\sum_{n}\left(<w_{n}\left|r^{2}\right| w_{n}>-<w_{n}|\mathbf{r}| w_{n}>^{2}\right) .
$$

If two interacting atoms, $A$ and $B$, are approximated[2] by coupled harmonic oscillators, the vdW energy correction can be taken to be the change of the zero-point energy of the coupled oscillations as the atoms approach; if only a single excitation frequency is associated to each atom, $\omega_{A}$, $\omega_{B}$, then

$$
E_{v d W}^{\text {London }}=-\frac{3 e^{4}}{2 m^{2}} \frac{Z_{A} Z_{B}}{\omega_{A} \omega_{B}\left(\omega_{A}+\omega_{B}\right)} \frac{1}{R_{A B}^{6}}
$$

where $Z_{A, B}$ is the total charge of $\mathrm{A}$ and $\mathrm{B}$, and $R_{A B}$ is the distance between the two atoms ( $e$ and $m$ are the electronic charge and mass). Now, adopting a simple classical theory of the atomic polarizability, the polarizability of an electronic shell of charge $e Z_{i}$ and mass $m Z_{i}$, tied to a heavy undeformable ion can be written as

$$
\alpha_{i} \simeq \frac{Z_{i} e^{2}}{m \omega_{i}^{2}} .
$$

Then, given the direct relation between polarizability and atomic volume[27], we assume that $\alpha_{i}=\gamma S_{i}^{3}$, where $\gamma$ is a proportionality constant, so that the atomic volume is expressed in terms of the MLWF spread, $S_{i}$. Rewriting eq. (2) in terms of the quantities defined above, one obtains an explicit, simple expression for the $C_{6} \mathrm{vdW}$ coefficient:

$$
C_{6}^{A B}=\frac{3}{2} \frac{\sqrt{Z_{A} Z_{B}} S_{A}^{3} S_{B}^{3} \gamma^{3 / 2}}{\left(\sqrt{Z_{B}} S_{A}^{3 / 2}+\sqrt{Z_{A}} S_{B}^{3 / 2}\right)} .
$$

The constant $\gamma$ can then be set up by imposing that the exact value for the $\mathrm{H}$ atom polarizability $\left(\alpha_{H}=4.5\right.$ a.u.) is obtained.

In order to achieve a better accuracy, one must properly deal with intrafragment MLWF overlap: in fact, the method is strictly valid for nonoverlapping fragments only; now, while the overlap between the MLWFs relative to separated fragments is usually negligible for all the fragment separation distances of interest, the same is not true for the MLWFs belonging to the same fragment, which and the excitation frequency (see eq. (3)), thus leading to a quantitative effect on the value of the $C_{6}$ coefficient. We take into account the effective change in volume due to intrafragment MLWF overlap by introducing a suitable reduction factor $\xi$ obtained by interpolating between the limiting cases of fully overlapping and non-overlapping MLWFs[24]. We therefore arrive at the following expression for the $C_{6}$ coefficient:

$$
C_{6}^{A B}=\frac{3}{2} \frac{\sqrt{Z_{A} Z_{B}} \xi_{A} S_{A}^{3} \xi_{B} S_{B}^{3} \gamma^{3 / 2}}{\left(\sqrt{Z_{B} \xi_{A}} S_{A}^{3 / 2}+\sqrt{Z_{A} \xi_{B}} S_{B}^{3 / 2}\right)},
$$

where $\xi_{A, B}$ represents the ratio between the effective and the free volume associated to the $A$-th and $B$-th MLWF[24].

Finally, in the previous DFT/vdW-WF2 method, the $\mathrm{vdW}$ interaction energy was computed as:

$E_{v d W}=-\sum_{i<j} f\left(R_{i j}\right) \frac{C_{6}^{i j}}{R_{i j}^{6}}=-\sum_{i<j} \frac{C_{6}^{i j}}{R_{i j}^{6}}+\sum_{i<j}\left(1-f\left(R_{i j}\right)\right) \frac{C_{6}^{i j}}{R_{i j}^{6}}$,

where $f\left(R_{i j}\right)$ is a short-range damping function, which is introduced not only to avoid the unphysical divergence of the vdW correction at small fragment separations, but also to eliminate double countings of correlation effects (in fact standard DFT approaches are able to describe short-range correlations); it is defined as:

$$
f\left(R_{i j}\right)=\frac{1}{1+e^{-a\left(R_{i j} / R_{s}-1\right)}} .
$$

The parameter $R_{s}$ represents the sum of the vdW radii $R_{s}=R_{i}^{v d W}+R_{j}^{v d W}$, with (by adopting the same criterion chosen above for the $\gamma$ parameter)

$$
R_{i}^{v d W}=R_{H}^{v d W} \frac{S_{i}}{\sqrt{3}}
$$

where $R_{H}^{v d W}$ is the literature[28] (1.20 $\AA$ ) vdW radius of the $\mathrm{H}$ atom, and, following Grimme et al.[29], $a \simeq 20$. Although $a$ is the only ad-hoc parameter of the method, while all the others are only determined by the basic information given by the MLWFs (that is from first principles calculations) and in many applications the results are only mildly dependent on the particular value of $a$, nonetheless, this parameter, together with the choice of a specific form of the above damping function, clearly imply a certain degree of empiricism.

In order to overcome this limitation, in the DFT/vdWWF2-x approach we replace the somehow artificial, shortrange damping function by a term that directly measures the quantum mechanical Pauli exchange repulsion between electronic orbitals and can be entirely expressed in terms of the MLWFs properties, without the need of introducing empirical parameters. Following ref. [30], using the dipole approximation for the Coulomb interaction, the exchange integral, for two closed electronic shells with total zero spin, is simply given by: 
where $q$ indicates the electronic charge of each electronic shell and $O$ is the overlap integral between the electronic shells, separated by $R$. In our specific case, assuming that an electronic orbital is described by the wave function relative to a quantum harmonic oscillator:

$$
\psi_{A}(r)=\left(\frac{3}{2 \pi}\right)^{3 / 4} \frac{1}{S_{A}^{3 / 2}} e^{-\left(\frac{3 r^{2}}{4 S_{A}^{2}}\right)},
$$

where $S_{A}$ is the spread of the corresponding MLWF, then one can easily obtain that :

$$
O_{A B}=8 \frac{S_{A}^{3} S_{B}^{3}}{\left(S_{A}^{2}+S_{B}^{2}\right)^{3}} e^{-\left(\frac{3}{2} \frac{R_{A B}^{2}}{S_{A}^{2}+S_{B}^{2}}\right)} .
$$

Then, the exchange integral can be expressed in terms of the MLWFs spreads as :

$$
J_{e x}^{A B}\left(R_{A B}\right)=\frac{q^{2} O_{A B}}{2 R_{A B}}=4 \frac{q^{2}}{R_{A B}} \frac{S_{A}^{3} S_{B}^{3}}{\left(S_{A}^{2}+S_{B}^{2}\right)^{3}} e^{-\left(\frac{3}{2} \frac{R_{A B}^{2}}{S_{A}^{2}+S_{B}^{2}}\right)} .
$$

Therefore, in the DFT/vdW-WF2-x method, the vdW interaction energy is computed as:

$$
E_{v d W}=-\sum_{i<j} \frac{C_{6}^{i j}}{R_{i j}^{6}}+\sum_{i<j} J_{e x}^{i j}\left(R_{i j}\right) .
$$

In this way the vdW energy correction is evaluated as the sum of two terms, both expressed in terms of the MLWfs spreads, thus making explicit the direct connection between attractive and repulsive parts of the vdW interaction[30].

Of course there are very weakly bonded systems, entirely dominated by $\mathrm{vdW}$ effects, where the repulsive term is not relevant for determining the equilibrium complex configuration. For instance, in the Ar-dimer case, DFT/vdW-WF2 and DFT/vdW-WF2-x predict the same equilibrium Ar-Ar distance and the same binding energy (within $0.1 \mathrm{meV}$ ). However, in most cases, a proper treatment of short-range repulsion is crucial to correctly describe the minimum, equilibrium configuration.

Applications of DFT/vdW-WF2-x to the case of Xe and water adsorbed on graphene have been performed with the Quantum-ESPRESSO ab initio package[31] and the MLWFs have been generated as a post-processing calculation using the WanT program[32], with ultrasoft pseudopotentials to describe the electron-ion interactions and taking PBE[33] as the reference, Generalized Gradient Approximation (GGA) DFT functional. PBE is chosen because it probably represents the most popular GGA functionals for standard DFT simulations of condensed-matter systems.

\section{Results}

In order to assess the accuracy of the DFT/vdWWF2-x method we first recall (in Table 1) the results
vdW-DF2[43], optB88-vdW[38], optB86b-vdW[39], vdW-DF-C09[40], vdW-DF-cx[41], rev-vdW-DF2[42], VV10[43], rVV10[44], PBE+TS-vdW[45, 47], PBE+MBD[45]. The errors are measured with respect to basis-set extrapolated $\operatorname{CCSD}(\mathrm{T})$ calculations of Takatani et al.[34]. Mean absolute relative errors (MARE in \%) and mean absolute errors (MAE in $\mathrm{kcal} / \mathrm{mol}$, and, in parenthesis, in $\mathrm{meV}$ ) are reported.

\begin{tabular}{|l|r|r|}
\hline method & MARE & MAE \\
\hline PBE & 55.5 & $2.56[111.0]$ \\
DFT/vdW-WF2 & 24.4 & $1.50[65.1]$ \\
DFT/vdW-WF2-x & 13.4 & $0.78[33.8]$ \\
\hline PBE-D3 & 11.4 & $0.50[21.7]$ \\
vdW-DF & 17.0 & $1.22[52.9]$ \\
vdW-DF2 & 14.7 & $0.94[40.8]$ \\
optB88-vdW & & $0.23[10.0]$ \\
optB86b-vdW & & $0.28[12.0]$ \\
vdW-DF-C09 & 5.0 & $0.31[13.4]$ \\
vdW-DF-cx & 7.0 & \\
rev-vdW-DF2 & 10.0 & $0.48[21.0]$ \\
VV10 & 4.4 & $0.31[13.4]$ \\
rVV10 & 4.3 & $0.30[13.0]$ \\
PBE+TS-vdW & 10.3 & $0.32[13.9]$ \\
PBE+MBD & 6.2 & $0.26[11.3]$ \\
\hline
\end{tabular}

obtained[24] by considering the S22 database of intermolecular interactions[25], a widely used benchmark database, consisting of weakly interacting molecules (a set of 22 weakly interacting dimers mostly of biological importance). Data are compared with reference binding energies calculated using high-level quantum chemical methods[34], with results obtained using the bare, non-vdW-corrected, PBE functional, and those obtained by other vdW-corrected DFT schemes, namely our previous scheme DFT/vdW-WF2[14], PBE-D3[29], vdWDF[35, 36], vdW-DF2[37], optB88-vdW[38], optB86bvdW[39], vdW-DF-C09[40], vdW-DF-cx[41], rev-vdWDF2[42], VV10[43] and rVV10[44] (the revised, computationally much more efficient version of the VV10 method), PBE+TS-vdW[45], and PBE+MBD[46].

As expected, considering the whole S22 database, pure PBE performs poorly and a substantial improvement can be obtained by vdW-corrected approaches. More importantly, the performances of the new DFT/vdWWF2-x scheme are clearly better than those of the previous DFT/vdW-WF2 method. In particular, the general tendency of DFT/vdW-WF2 to overbind is considerably reduced by DFT/vdW-WF2-x. Interestingly, with DFT/vdW-WF2-x the mean absolute error (MAE), 0.78 $\mathrm{kcal} / \mathrm{mol}$, is well below the so-called "chemical accuracy" threshold of $1 \mathrm{kcal} / \mathrm{mol}$, required to attribute a genuine quantitative character to the predictions of an ab initio scheme. Moreover, DFT/vdW-WF2-x performs better than the more sophisticated vdW-DF and vdW-DF2 methods, based on the use of a nonlocal expression for the correlation energy-term, is comparable, as far as the 
optB86b-vdW, vdW-DF-C09, vdW-DF-cx, rev-vdW-DF2, rVV10, VV10, PBE+TS-vdW, and PBE+MBD schemes, which are among the most accurate, presently available, vdW-corrected DFT approaches for noncovalently bound complexes[38-42, 44, 46].

It is certainly important to test the applicability of the DFT/vdW-WF2-x method also to extended systems, which of course are of particular interest because, in this case, higher-quality chemistry methods are typically too computationally demanding. We have therefore considered[24] the adsorption of a single Ar atom on graphite, that is a typical physisorption process, and also the cases of a Xe atom and a water molecule interacting with graphene, which are adsorption processes characterized by a little stronger interactions with the substrate. Calculations have been performed using the same DFT approach followed in ref. [20] and considering the adsorption on the most favored hollow sites only, illustrated in Figure 1 for the case of water on graphene (with the two $\mathrm{OH}$ bonds pointing down to graphene).

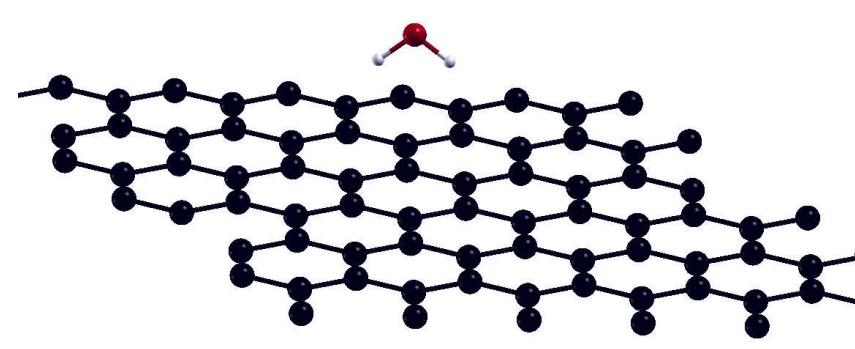

Figure 1. Water molecule on graphene in the most favored adsorption configuration.

Tables 2 and 3 report the binding energy, $\mathrm{E}_{b}$, and equilibrium distance, $\mathrm{R}$, for Ar-graphite, Xe-graphene, and water-graphene. Data obtained at the PBE, DFT/vdWWF2, and DFT/vdW-WF2-x level are compared to reference theoretical and experimental estimates (the latter relative to adsorptions on graphite, in the absence of specific experimental data on graphene).

As can be seen, in all the cases the pure PBE functional largely underbinds while vdW-corrected schemes predict much larger binding energies and shorter equilibrium distances. Moreover, DFT/vdW-WF2-x represents an evident improvement compared to DFT/vdW-WF2, in line with what previously observed in the application to the S22 database, showing again that, at equilibrium distances, the effect of the repulsive term is significant. In particular, for Ar-graphite the DFT/vdW-WF2-x binding energy (-115 $\mathrm{meV}$ ) is very close to the values reported by Tkatchenko et al. [48] (-116 meV) and Bichoutskaia and Pyper[49] $(-111 \mathrm{meV})$. Moreover, this value is also compatible with one of the few experimental estimates, represented by the
[50-52], for Xe-graphite refs. [53, 54], for water-graphene refs. [55, 56]) and experimental (for Ar-graphite refs. [50, 57], for

Xe-graphite refs. [50, 54], for water-graphite refs. [58]) reference data.

\begin{tabular}{|l|l|c|}
\hline system & method & $\mathrm{E}_{b}(\mathrm{meV})$ \\
\hline Ar-graphite & PBE & -12 \\
Ar-graphite & DFT/vdW-WF2 & -145 \\
Ar-graphite & DFT/vdW-WF2-x & -115 \\
Ar-graphite & ref.theory & $-116<->-111$ \\
Ar-graphite & ref.expt. & -99 \\
\hline Xe-graphene & PBE & -11 \\
Xe-graphene & DFT/vdW-WF2 & -266 \\
Xe-graphene & DFT/vdW-WF2-x & -170 \\
Xe-graphene & ref.theory & $-180<->-143$ \\
Xe-graphite & ref.theory & $-180<->-166$ \\
Xe-graphite & ref.expt & $-170<->-160$ \\
\hline $\mathrm{H}_{2} \mathrm{O}$-graphene & PBE & -26 \\
$\mathrm{H}_{2} \mathrm{O}$-graphene & DFT/vdW-WF2 & -171 \\
$\mathrm{H}_{2} \mathrm{O}$-graphene & DFT/vdW-WF2-x & -161 \\
$\mathrm{H}_{2} \mathrm{O}$-graphene & ref.theory & $-140<->-72$ \\
$\mathrm{H}_{2} \mathrm{O}$-graphite & ref.expt & -156 \\
\hline
\end{tabular}

Table 3. Equilibrium distance R, for an Ar atom adsorbed on graphite, and $\mathrm{Xe}$ and water on graphene, compared to available theoretical (for Ar-graphite refs. [48, 49], for Xe-graphene refs. [50-52], for Xe-graphite refs. [53, 54], for water-graphene refs. $[55,56]$ ) and experimental (for Ar-graphite refs. [50, 57], for

Xe-graphite refs. [50, 54], for water-graphite refs. [58]) reference data.

\begin{tabular}{|l|l|c|}
\hline system & method & $\mathrm{R}(\AA)$ \\
\hline Ar-graphite & PBE & 4.0 \\
Ar-graphite & DFT/vdW-WF2 & 3.3 \\
Ar-graphite & DFT/vdW-WF2-x & 3.5 \\
Ar-graphite & ref.theory & 3.3 \\
Ar-graphite & ref.expt. & $3.1<->3.2$ \\
\hline Xe-graphene & PBE & 4.4 \\
Xe-graphene & DFT/vdW-WF2 & 3.4 \\
Xe-graphene & DFT/vdW-WF2-x & 4.0 \\
Xe-graphene & ref.theory & $2.7<->3.7$ \\
Xe-graphite & ref.theory & - \\
Xe-graphite & ref.expt & 3.6 \\
\hline $\mathrm{H}_{2} \mathrm{O}$-graphene & PBE & 3.7 \\
$\mathrm{H}_{2} \mathrm{O}$-graphene & DFT/vdW-WF2 & 3.1 \\
$\mathrm{H}_{2} \mathrm{O}$-graphene & DFT/vdW-WF2-x & 3.2 \\
$\mathrm{H}_{2} \mathrm{O}$-graphene & ref.theory & $3.3<->3.5$ \\
$\mathrm{H}_{2} \mathrm{O}$-graphite & ref.expt & - \\
\hline
\end{tabular}

measurement of the latent heat of condensation relative to the adsorption of an Ar monolayer on graphite: $-119 \pm 2$ $\mathrm{meV} /$ atom[59]. It is also close to the "best estimate" (obtained from a combination of experimental and theoretical, mainly semiempirically-based, data) reported in the milestone review paper by Vidali et al.[50], that is $-99 \pm$ $4 \mathrm{meV}$. Our Ar-graphite equilibrium distance computed by DFT/vdW-WF2-x is instead somehow larger than that reported in other theoretical studies[48, 49] (3.3 $)$, and also 
ref. [57]). However one sould observe that an accurate estimate of this quantity is more difficult due to the relatively shallow potential energy curve of this system.

Concerning the interaction of Xe with graphene, previous theoretical calculations exist (see Tables 2 and 3); in particular, the MP2 method[51] gives an estimate of -143 meV for the binding energy at a separation of the Xe atom from the substrate of $3.6 \AA$. Our DFT/vdW-WF2-x result of $-170 \mathrm{meV}$, is not far from the MP2 value also considering that the MP2 results were not directly obtained from a calculation on an infinite graphene sheet, as in the present approach, but were instead derived by an extrapolation procedure using calculations on Xe interacting with polycylic aromatic hydrocarbons such as coronene. Moreover, the DFT/vdW-WF2-x binding energy agrees well with the "best estimate" $(-162 \pm 4 \mathrm{meV})$ reported by Vidali et al. [50] for Xe on graphite. Instead the binding energy obtained by the previous DFT/vdW-WF2 scheme turns out to be evidently overestimated, and the same conclusion applies by referring to the experimental reference range too (see Table 2). With respect to the reference, theoretical and experimental estimates for the equilibrium distance, our DFT/vdW-WF2-x value of $4.0 \AA$ appears to be significantly larger, but, for this quantity, the same considerations made above for Ar-graphite apply.

Finally, as far as the adsorption of a water molecule on graphene/graphite is concerned, the experimental estimates of the binding energy are scarce[26]: for watergraphite an experimental estimate of $-156 \mathrm{meV}$ exist, which is very close to our DFT/vdW-WF2-x value, while interaction energies in the range from -65 to $-97 \mathrm{meV}$ were obtained from empirical force-field simulations[60], aimed at reproducing the experimentally observed contact angles of water droplets on graphite. From the theoretical point of view, highly accurate ab initio data (coupled clusters at the complete basis set limit) are currently limited to the water-benzene system[61] and other quantum-chemistry estimates are quite scattered and based on extrapolations of the interaction energies calculated for polycyclic aromatic hydrocarbons of increasing size. A DFT/coupled cluster method (DFT/CC) has been applied to evaluate the electronic binding energy of a water molecule with graphite and graphene, giving as a result, $-155 \mathrm{meV}$ at $3.24 \AA$ and $-138 \mathrm{meV}$ at $3.26 \AA$, respectively[26]. Also in this case DFT/vdW-WF2-x performs well: not only the binding energy but even the equilibrium distance are close to the reference values, although the improvement with respect to the previous DFT/vdWWF2 method is smaller than for Ar-graphite and Xegraphene. This is probably because the water-graphene interaction is not entirely due to vdW forces but results from a complex interplay of hydrogen bonding, electrostatic, and vdW effects.

\section{Conclusions}

In summary, we have described the DFT/vdW-WF2-x method for including van der Waals (vdW) interactions previous DFT/vdW-WF2 method, replaces the empirical, short-range, damping function by an estimate of the Pauli exchange repulsion, also obtained by the MLWFs properties. Applications to systems contained in the popular S22 molecular database and to the case of adsorption of $\mathrm{Ar}$ on graphite, and $\mathrm{Xe}$ and water on graphene, indicate that the new method, besides being more physically founded, represents a systematic improvement in the description of systems where vdW interactions play a significant role.

\section{Acknowledgements}

We acknowledge funding from Fondazione Cariparo, Progetti di Eccellenza 2017, relative to the project: "Engineering van der Waals Interactions: Innovative paradigm for the control of Nanoscale Phenomena".

\section{References}

[1] See, for instance, W. Kohn, D. E. Makarov, Phys. Rev. Lett. 80, 4153 (1998).

[2] R. Eisenhitz and F. London, Z. Phys. 60, 491 (1930).

[3] K. E. Riley, M. Pitoňák, P. Jurečka, P. Hobza, Chem. Rev. 110, 5023 (2010).

[4] A. Tkatchenko, L. Romaner, O. T. Hofmann, E. Zojer, C. Ambrosch-Draxl, and M. Scheffler, MRS Bulletin, 35, 435 (2010).

[5] J. Klimeš, A. Michaelides, J. Chem. Phys. 137, 120901 (2012).

[6] L. M. Woods, D. A. R. Dalvit, A. Tkatchenko, P. Rodriguez-Lopez, A. W. Rodriguez, R. Podgornik, Rev. Mod. Phys. 88, 045003 (2016).

[7] S. Grimme, A. Hansen, J. G. Brandenburg, C. Bannwarth, Chem. Rev. 116, 5105 (2016).

[8] J. Hermann, R. A. DiStasio Jr., A. Tkatchenko, Chem. Rev. 117, 4714 (2017).

[9] K. Berland, V. R. Cooper, K. Lee, E. Schröder, T. Thonhauser, P. Hyldgaard, B. I. Lundqvist, Rep. Prog. Phys. 78, 066501 (2015).

[10] N. Marzari and D. Vanderbilt, Phys. Rev. B 56, 12847 (1997).

[11] P. L. Silvestrelli, Phys. Rev. Lett 100, 053002 (2008).

[12] P. L. Silvestrelli, J. Phys. Chem. A 113, 5224 (2009).

[13] L. Andrinopoulos, N. D. M. Hine, A. A. Mostofi, J. Chem. Phys. 135, 154105 (2011).

[14] A. Ambrosetti, P. L. Silvestrelli, Phys. Rev. B 85, 073101 (2012); Phys. Rev. B 87, 039902 (2013).

[15] P. L. Silvestrelli and A. Ambrosetti, Phys. Rev. B 87, 075401 (2013).

[16] P. L. Silvestrelli, J. Chem. Phys. 139, 054106 (2013).

[17] P. L. Silvestrelli, K. Benyahia, S. Grubisic, F. Ancilotto, and F. Toigo, J. Chem. Phys. 130, 074702 (2009).

[18] P. L. Silvestrelli, Chem. Phys. Lett. 475, 285 (2009).

[19] P. L. Silvestrelli, F. Toigo, F. Ancilotto, J. Phys. Chem. C 113, 17124 (2009). 
[21] P. L. Silvestrelli, A. Ambrosetti, S. Grubisiê, and F. Ancilotto, Phys. Rev. B 85, 165405 (2012).

[22] F. Costanzo, P. L. Silvestrelli, Francesco Ancilotto, J. Chem. Theory Comp. 8, 1288 (2012).

[23] A. Ambrosetti, F. Ancilotto, P. L. Silvestrelli, J. Phys. Chem. C 117, 321 (2013).

[24] P. L. Silvestrelli, A. Ambrosetti, J. Chem. Phys. 150, 164109 (2019).

[25] P. Jurečka, J. Šponer, J. Černy, P. Hobza, Phys. Chem. Chem. Phys. 8, 1985 (2006).

[26] M. Rubeš, P. Nachtigall, J. Vondrášek, O. Bludský, J. Phys. Chem. C 113, 8412 (2009).

[27] T. Brink, J. S. Murray, P. Politzer, J. Chem. Phys. 98, 4305 (1993).

[28] A. Bondi, J. Phys. Chem. 68, 441 (1964).

[29] S. Grimme, J. Antony, T. Schwabe, C. MückLichtenfeld, Org. Biomol. Chem. 5, 741 (2007); S. Grimme, J. Antony, S. Ehrlich, H. Krieg, J. Chem. Phys. 132, 154104 (2010).

[30] D. V. Fedorov, M. Sadhukhan, M. Stöd, A. Tkatchenko, Phys. Rev. Lett. 121, 183401 (2018).

[31] P. Giannozzi, S. Baroni, N. Bonini, M. Calandra, R. Car, C. Cavazzoni, D. Ceresoli, G. L. Chiarotti, M. Cococcioni, I. Dabo, A. Dal Corso, S. Fabris, G. Fratesi, S. de Gironcoli, R. Gebauer, U. Gerstmann, C. Gougoussis, A. Kokalj, M. Lazzeri, L. Martin-Samos, N. Marzari, F. Mauri, R. Mazzarello, S. Paolini, A. Pasquarello, L. Paulatto, C. Sbraccia, S. Scandolo, G. Sclauzero, A. P. Seitsonen, A. Smogunov, P. Umari, R. M. Wentzcovitch, J.Phys.: Condens. Matter 21, 395502 (2009); http://arxiv.org/abs/0906.2569.

[32] WanT code by A. Ferretti, B. Bonferroni, A. Calzolari, and M. Buongiorno Nardelli, (http://www.wannier-transport.org). See also: A. Calzolari, N. Marzari, I. Souza and M. Buongiorno Nardelli, Phys. Rev. B 69, 035108 (2004).

[33] J.P. Perdew, K. Burke, M. Ernzerhof, Phys. Rev. Lett. 77, 3865 (1996).

[34] T. Takatani, E. G. Hohenstein, M. Malagoli, M. S. Marshall, C. D. Sherril, J. Chem. Phys. 132, 144104 (2010).

[35] M. Dion, H. Rydberg, E. Schröder, D. C. Langreth, B. I. Lundqvist, Phys. Rev. Lett. 92, 246401 (2004); G. Roman-Perez, J. M. Soler, Phys. Rev. Lett. 103, 096102 (2009).

[36] T. Thonhauser, V. R. Cooper, S. Li, A. Puzder, P. Hyldgaard, D. C. Langreth, Phys. Rev. B 76, 125112 (2007).

[37] K. Lee, É. D. Murray, L. Kong, B. I. Lundqvist, and D. C. Langreth, Phys. Rev. B 82, 081101(R) (2010).
[39] J. Klimes, D. R. Bowler, A. Michaelides, Phys. Rev. B 83, 195131 (2011).

[40] V. R. Cooper, Phys. Rev. B 81, 161104(R) (2010).

[41] K. Berland, P. Hyldgaard, Phys. Rev. B 89, 035412 (2014).

[42] I. Hamada, Phys. Rev. B 89, 121103(R) (2014).

[43] O. A. Vydrov, T. van Voorhis, J. Chem. Phys. 133, 244103 (2010).

[44] R. Sabatini, T. Gorni, S. de Gironcoli, Phys. Rev. B 87, 041108(R) (2013).

[45] A. Tkatchenko, M. Scheffler, Phys. Rev. Lett. 102, 073005 (2009).

[46] A. Tkatchenko, R. A. Di Stasio, R. Car, M. Scheffler, Phys. Rev. Lett. 108, 236402 (2012).

[47] R. A. Di Stasio Jr., O. A. von Lilienfeld, A. Tkatchenko, PNAS 109, 14791 (2012).

[48] A. Tkatchenko, O. A. von Lilienfeld, Phys. Rev. B 73, 153406 (2006).

[49] E. Bichoutskaia, N. C. Pyper, J. Chem. Phys. 128, 024709 (2008).

[50] G. Vidali, G. Ihm, H. Y. Kim, M. W. Cole, Surf. Sci. Rep. 12, 133 (1991).

[51] L. Sheng, Y. Ono, T. Taketsugu, J. Phys. Chem. C 114, 3544 (2010).

[52] I. A. Pašti, A. Jovanović, A. S. Dobrota, S. V. Mentus, B. Johansson, N. V. Skorodumova, Appl. Surf. Sci. 436, 433 (2018).

[53] M. P. de Lara-Castells, M. Bartolomei, A. O. Mitrushchenkov and H. Stoll, J. Chem. Phys. 143, 194701 (2015).

[54] D. L. Chen, W. A. S. L. Mandeltort, J. T. Yates Jr., M. W. Cole, and J. K. Johnson, Phys. Rev. Lett. 110, 135503 (2013).

[55] P. L. Silvestrelli, A. Ambrosetti, J. Chem. Phys. 140, 124107 (2014).

[56] J. G. Brandenburg, A. Zen, M. Fitzner, B. Ramberger, G. Kresse, T. Tsatsoulis, A. Grüneis, A. Michaelides, and D. Alfé, J. Phys. Chem. Lett. 10, 358 (2019).

[57] Chemistry and Physics of Solid Surfaces IV (R. Vanselow and R. Howe, Springer Series in Chemical Physics, 1982).

[58] Chemistry and Physics of Carbon (N. N. Avgul, A. V. Kieslev, Dekker, New York, 1970).

[59] C. G. Shaw, S. C. Fain Jr., Surf. Sci. 91, L1 (1980).

[60] T. Werder et al., J. Phys. Chem. B 107, 1345 (2003).

[61] D. Feller, J. Phys. Chem. A 103, 7558 (1999); S. Tsuzuki et al., J. Am. Chem. Soc. 122, 11450 (2000); P. Jurecka et al., Phys. Chem. Chem. Phys. 8, 1985 (2000). 